\title{
Boodlea composita (Harv.) F.Brand (Chlorophyta) no litoral nordeste do Brasil ${ }^{1}$
}

Aigara Miranda Alves ${ }^{2,4}$, Lísia Mônica de Souza Gestinari³ ${ }^{3}$ Neilton Argolo de Andrade², Wellington Romualdo de Almeida ${ }^{2}$ e Carlos Wallace do Nascimento Moura ${ }^{2}$

Recebido em 25/05/2011. Aceito em 12/04/2012

\begin{abstract}
RESUMO
(Boodlea composita (Harv.) F.Brand (Chlorophyta) no litoral nordeste do Brasil). Este estudo relata a primeira ocorrência de Boodlea composita (Harv.) F.Brand (Chlorophyta) para o litoral nordeste do Brasil e o segundo registro para o litoral brasileiro. O material foi coletado ao longo do litoral da Bahia ( $08^{\circ} 20^{\prime} 07^{\prime \prime}-18^{\circ} 20^{\prime} 07^{\prime \prime} \mathrm{S}$ e $\left.30^{\circ} 20^{\prime} 37^{\prime \prime}-46^{\circ} 36^{\prime} 59^{\prime \prime} \mathrm{W}\right)$. A distribuição de B. composita foi ampliada no litoral do Brasil, uma vez que esta era conhecida apenas para a região sudeste, especificamente para Cabo Frio, Rio de Janeiro. No Atlântico Americano, B. composita apresenta limite norte de distribuição nas Bermudas e limite sul, no Rio de Janeiro.
\end{abstract}

Palavras-chave: Bahia, Boodlea, Boodleaceae, Chlorophyta, Litoral Nordeste do Brasil

\begin{abstract}
(Boodlea composita (Harv.) F.Brand (Chlorophyta) in the northeastern coast of Brazil). This study reports the first occurrence of Boodlea composita (Harv.) F.Brand (Chlorophyta) to the northeastern coast of Brazil and the second record for the Brazilian coast. The material was collected along the coast of Bahia $\left(08^{\circ} 20^{\prime} 07^{\prime \prime}-18^{\circ} 20^{\prime} 07^{\prime \prime} \mathrm{S}\right.$ and $30^{\circ} 20^{\prime} 37^{\prime \prime}$ $\left.46^{\circ} 36^{\prime} 59^{\prime \prime} \mathrm{W}\right)$. The distribution of B. composita was expanded along the coast of Brazil, since this was known only to the Southeast, specifically to Cabo Frio, Rio de Janeiro. In American Atlantic, B. composita presents northern limit of distribution in Bermuda and southern limit at Rio de Janeiro.
\end{abstract}

Key words: Bahia, Boodlea, Boodleaceae, Chlorophyta, northeastern coast of Brazil

\section{Introdução}

O gênero Boodlea (Dickie) G.Murray \& De Toni foi descrito por Murray \& De Toni (Murray 1889) para acomodar a espécie Cladophora coacta descrita por Dickie (1876). Boodlea é caracterizado por apresentar talo composto por ramos septados, unisseriados, formando lâmina reticulada em vários planos com células tenaculares unindo ramos adjacentes. O gênero Boodlea foi incluído na família Boodleaceae por Børgesen (1925), juntamente com o gênero Cladophoropsis Børgesen, baseado no atraso da formação da parede celular na base dos ramos. Boodleaceae foi redefinida por Egerod (1952), a qual foi caracterizada por apresentar talo formando lâmina reticulada ou almofadas compostas de filamentos com ramificação oposta e anastomosados por células tenaculares formadas no ápice das células apicais.

Boodlea possui distribuição pantropical, ocorrendo nos oceanos Atlântico, Pacífico e Índico. Guiry \& Guiry (2011) reconhecem seis espécies válidas para o gênero. Duas destas, Boodlea composita (Harv.) F.Brand e B. struveoides M. Howe foram referidas para o Atlântico Americano (Wynne 2011).

Atualmente no Brasil o gênero está representado por $B$. composita, a qual era conhecida apenas para o litoral sudeste brasileiro, a partir de material coletado em Cabo Frio, Rio de Janeiro (Yoneshigue et al. 1986).

Este estudo refere-se ao primeiro registro de Boodlea composita para o litoral nordeste do Brasil e ao segundo para o litoral brasileiro. São descritas características morfo-

\footnotetext{
Parte da dissertação de Mestrado da primeira Autora

2 Universidade Estadual de Feira de Santana, Laboratório de Ficologia, Feira de Santana, BA, Brasil

3 Universidade Federal do Rio de Janeiro, Núcleo em Ecologia e Desenvolvimento Socioambiental de Macaé, Macaé, RJ, Brasil

4 Autor para correspondência: aigarama@yahoo.com.br
} 
lógicas de espécimes de B. composita registrados no litoral do estado da Bahia.

\section{Material e métodos}

O Estado da Bahia, situado entre as coordenadas $08^{\circ} 20^{\prime} 07^{\prime \prime}-18^{\circ} 20^{\prime} 07^{\prime \prime} S$ e $30^{\circ} 20^{\prime} 37^{\prime \prime}-46^{\circ} 36^{\prime} 59^{\prime \prime} \mathrm{W}$, possui o litoral mais extenso do Brasil, com $1.103 \mathrm{~km}$ e apresenta uma grande diversidade de ambientes costeiros, como praias arenosas, recifes de coral, formações de arenito, costões rochosos e manguezais (Nunes \& Guimarães 2008).

Os espécimes estudados foram coletados ao longo do litoral baiano. Os vouchers estão depositados no Herbário da Universidade Estadual de Feira de Santana (HUEFS). Em adição foram analisados espécimes coletados em Cabo Frio, Rio de Janeiro, da coleção da Dra. Yocie Yoneshigue Valentin depositados no Herbário da Universidade Federal do Rio de Janeiro (RFA).

As características morfológicas e anatômicas dos espécimes de Boodlea composita foram analisadas com o auxílio de estereomicroscópio e microscópio fotônico acoplado com ocular micrométrica e camêra fotográfica digital. As dimensões (diâmetro e comprimento) de cada estrutura foram estabelecidas através de 10 a 20 medidas feitas ao acaso em cada espécime.

A natureza química das inclusões cristalinas observadas foi determinada através de testes de solubilidade química, expondo estas a ácido clorídrico $(\mathrm{HCl}) 1 \mathrm{~N}$, hipoclorito de sódio $(\mathrm{NaClO})$ a $5 \%$, ácido acético $\left(\mathrm{C}_{2} \mathrm{H}_{4} \mathrm{O}_{2}\right)$ aquoso a $67 \%$ (Yasue 1969; Lelliaert \& Coppejans 2004; Alves et al. 2009, 2010, 2011) e ácido fluorídrico (HF) a 40\%, modificado de Faegri \& Iversen (1975). A presença ou ausência de birrefringência das inclusões foi avaliada através de microscópio de contraste de interferência (Nomarski).

\section{Resultados e discussão}

Boodlea composita (Harv.) F.Brand, Beih., Bot. Centralbl. 18(1): 165-193, pl. 6, fig. 28-35. 1904.

Fig. 1 A-X

Talo verde claro, prostrado a ereto, formando densas almofadas, composto de filamentos ramificados, fixo ao substrato por rizoides ramificados originados da base da célula do estipe de talos jovens, por rizoides produzidos no ápice das células apicais e por células tenaculares, formadas em qualquer parte do talo. Ramificação oposta à unilateral; eixo principal apresentando ramificação oposta na base a irregular na região mediano-apical. Ramos laterais com atraso na formação dos septos, os quais permanecem conectados à célula parental. Divisão celular por invaginação centrípeta da parede; ocasionalmente ocorre divisão segregativa, devido a dano celular. Reforço do talo por denso entrelaçamento de ramos curvos e anastomoses de células adjacentes por células tenaculares de dois tipos (do tipo 1 - células hapteroidais formadas no ápice das células e do tipo 2 - células hapteroidais formadas lateralmente entre células adjacentes). Células multinucleadas com numerosos cloroplastos discoides, unidos formando retículo parietal, com único pirenoide cada. Célula apical cilíndrica a levemente achatada com ápice arredondado, reto ou curvado, medindo 380-(1198)-11360 $\mu \mathrm{m}$ de compr. x 100-(128)-160 $\mu \mathrm{m}$ de diâm.; células do eixo principal cilíndricas, com 300-(592)-1300 $\mu$ m de compr. x 90-(135)$200 \mu \mathrm{m}$ de diâm.; parede celular fina com menos de 2,5 $\mu \mathrm{m}$ de espessura. Cristais birrefringentes de oxalato de cálcio, em forma de hexágonos alongados a finas agulhas agrupadas, presentes em todas as células do talo, exceto nas células tenaculares. Células férteis com protoplasto dividido, com estruturas reprodutivas liberadas por papilas laterais e apicais. Talos férteis encontrados nos meses de março e junho.

A espécie é pouco comum na área de estudo, crescendo sobre substrato rochoso, formando tufos densos, em locais protegidos ou expostos à arrebentação das ondas. Foi encontrada crescendo junto à Anadyomene stellata (Wulfen in Jacq.) C.Agardh, Phyllodictyon anastomosans (Harv.) Kraft \& M.J.Wynne, Dictyosphaeria versluysii Weber Bosse, Chaetomorpha brachygona Harv., Caulerpa fastigiata Mont., Caulerpella ambigua (Okamura) Prud'homme \& Lokhorst, Cladophora vagabunda (L.) C.Hoek, Bryopsis pennata J.V.Lamour., Cladophoropsis membranacea (H.Bang ex C.Agardh) Børgesen, Boodleopsis pusilla (F.S.Collins) W.R.Taylor, A.B.Joly \& Bernat., Dictyopteris delicatula J.V.Lamour., Dictyota sp., Amphiroa anastomosans Weber Bosse, A. fragilissima (L.) J.V.Lamour., Digenea simplex (Wulfen) C.Agardh, Jania adhaerens J.V.Lamour., Hypnea musciformis (Wulfen) J.V.Lamour. e Palisada perforata (Bory de Saint-Vincent) K.W. Nam. Teve como epífitas Erythrotrichia carnea (Dillwyn) J.Agardh, Sahlingia subintegra (Rosenv.) Kornmann, calcárias crostosas e diatomáceas.

Material analisado: BRASIL. Bahia: Sítio do Conde, 20/ IV/2007, Alves, A.M. \& Santana, C.S. s.n. (HUEFS 136300); Salvador, Praia da Pituba, 09/VIII/2006, Alves, A.M. s.n. (HUEFS 136301), Praia da Barra, 23-II-2008, Almeida, W.R. s.n. (HUEFS 136665); Ilha de Itaparica, Vera Cruz, Praia da Coroa, 07/X/1994, Moura, C.W.N. s.n. (HUEFS 136302), Praia da Conceição, 18/III/2007, Moura, C.W.N. et al. s.n. (HUEFS 136303), 18/V/2007, Alves, A.M. et al. s.n. (HUEFS 136304), 14/VII/2007, Moura, C.W.N. et al. s.n. (HUEFS 136305), Praia da Enseada do Pedrão, 19/III/2007, Moura, C.W.N. et al. s.n. (HUEFS 136306); Ilhéus, Praia do Aeroporto, 08/IX/2006, Alves, A.M. et al. s.n. (HUEFS 136307); Mucuri, Praia da Costa Dourada, 12/VI/2007, Alves, A.M. \& Santana, C.S. s.n. (HUEFS 136308).

Material adicional analisado: BRASIL. Rio de Janeiro: Ponta Pai Vitório, Mesolitoral, 28/VII/1983, Yoneshigue, 

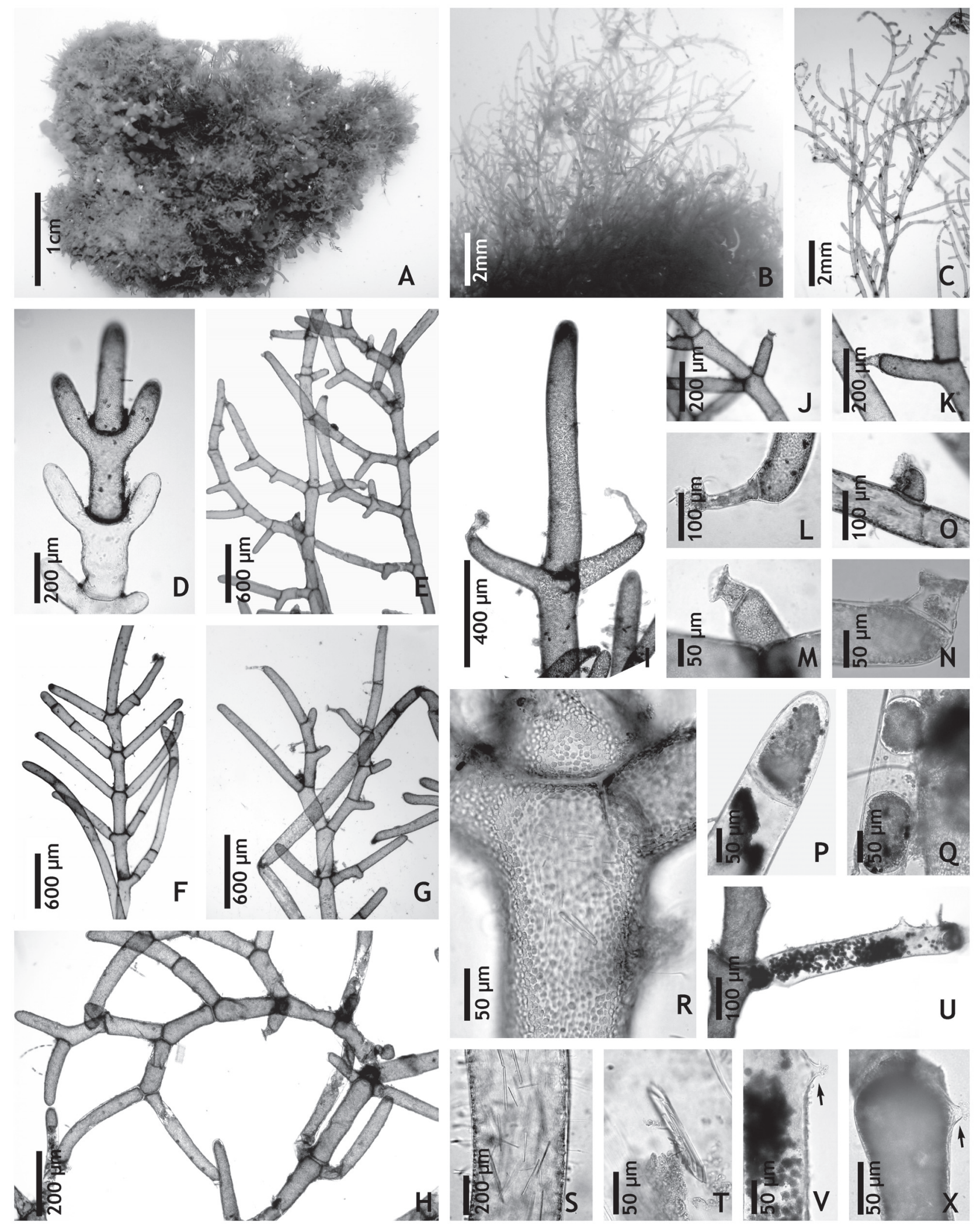

Figura 1. Boodlea composita (Harv.) F.Brand. A-B. Aspecto geral. C-H. Detalhe da ramificação do talo. C, E, G-H. Ramificação oposta no eixo principal a unilateral nos eixos secundários. D, F. Ramificação oposta. I. Célula apical alongada. J-M. Detalhe de células tenaculares do tipo 1. O. Célula tentacular do tipo 2. P. Célula apical jovem. Q. Massas citoplamáticas formadas por divisão celular segregativa. R. Detalhe dos cloroplastos formando retículo. S-T. Cristais de oxalato de cálcio em forma de hexágonos alongados. U. Ramo com célula fértil. V-X. Célula fértil com papila. 
Y., s.n. YY 4010H (UFRJ-RFA); 18/VII/1981, Yoneshigue, Y. s.n. MOF 352 (UFRJ-RFA); 18/VII/1981, Yoneshigue, Y. s.n. YY 2831 (UFRJ-RFA).

O material analisado para o litoral baiano concorda com as descrições e ilustrações fornecidas por Børgesen (1913, 1930), Egerod (1952), Meñez (1961), Yoneshigue et al. (1986), Littler \& Littler (2000) e Leliaert \& Coppejans (2007).

Yoneshigue et al. (1986) também encontraram $B$. composita crescendo no mesmo habitat de Cladophoropsis membranacea, os quais podem ser facilmente confundidos em campo.

Baseados em distâncias imunológicas Olsen-Stojkovich et al. (1986) demonstraram que Boodlea composita, Phyllodictyon anastomosans, Cladophoropsis membranacea e Chamaedoris peniculum (J.Ellis \& Sol.) Kuntze formavam um clado altamente associado. Estudos filogenéticos posteriores, baseados em análises de sequências de SSU e LSU rDNA (Leliaert \& Coppejans 2007; Leliaert et al. 2007a, 2007b, 2007c), LSU rRNA (Leliaert et al. 2003) e $18 \mathrm{~S}$ rRNA (Bakker et al. 1994), confirmaram os dados de Olsen-Stojkovich et al. (1986) e revelaram uma extrema relação entre os gêneros Boodlea, Chamaedoris Mont., Cladophoropsis, Phyllodictyon J.E.Gray, Struvea Sond. e Struveopsis Rhyne \& H.Rob.

Leliaert \& Coppejans (2007), estudando as variações morfológicas dos talos de espécies de Boodlea, Phyllodictyon, Cladophoropsis, Nereodictyon Gerloff e Struveopsis, consideraram estes gêneros como parte do complexo de espécies Boodlea composita-Phyllodictyon anastomosans. Para este complexo, os autores reconheceram sete morfótipos (anastomosans, composita, delicatula, kenyensis, montagnei, siamensis e struveopsis). O material coletado no litoral da Bahia assemelha-se ao morfótipo siamensis, por apresentar talo com ramificação irregular, unilateral nas regiões distais e oposta nas regiões basais, abundância de células tenaculares e dimensões celulares similares.

Os cristais de oxalato de cálcio observados nas células dos espécimes analisados correspondem àqueles observados por Leliaert \& Coppejans (2004, 2007). Segundo estes autores, a morfologia destes cristais parece ser espécie ou gênero específico, indicando que o seu desenvolvimento é geneticamente controlado e, portanto, com valor sistemático.

A partir deste estudo a distribuição de B. composita foi ampliada no litoral brasileiro, uma vez que a espécie era conhecida apenas para a região sudeste, especificamente para Cabo Frio, Rio de Janeiro.

\section{Agradecimentos}

Os autores agradecem à FAPESB (Proc. PPP 0011/2006), pelo financiamento parcial do projeto, à CAPES, pela bolsa concedida primeira autora, à UEFS pela infraestrutura disponibilizada e auxílios. À profa Dra. Yocie Yoneshigue
Valentin, da Universidade Federal do Rio de Janeiro, pelo acesso ao material adicional analisado.

\section{Referências bibliográficas}

Alves, A.M.; C.W.N. Moura; G.L. Alves \& L.M.S. Gestinari. 2009. Os gêneros Chaetomorpha Kütz. nom. cons. e Rhizoclonium Kütz. (Chlorophyta) do litoral do Estado da Bahia, Brasil. Revista Brasileira de Botânica 32: 545-570.

Alves, A.M.; L.M.S. Gestinari \& C.W.N. Moura. 2010. La familia Valoniaceae (Chlorophyta) en el estado de Bahía, Brasil: aspectos morfológicos y de distribución. Hidrobiológica 20: 171-184.

Alves, A.M.; L.M.S. Gestinari \& C.W.N. Moura. 2011. Morphology and taxonomy of Anadyomene species (Cladophorales, Chlorophyta) from Bahia, Brazil. Botanica Marina 54: 135-145.

Bakker, F.T.; Olsen, J.L.; Stam, W.T. \& van den Hoek, C. 1994. The Cladophora complex (Chlorophyta): new views based on the 18S rRNA gene sequences. Molecular Phylogenetics and Evolution 3(4): 365-382.

Børgesen, F. 1913. The marine algae of the Danish West Indies. 1. Chlorophyceae. Dansk Botanisk Arkiv 1(4): 1-158.

Børgesen, F. 1925. Marine algae from the Canary Islands especially Teneriffe and GranCanaria. I. Chlorophyceae. Kongelige Danske Videnskabernes Selskab, Biologiske Meddelelser 5(3): 1-123.

Børgesen, F. 1930. Some Indian green and brown algae especially from the shores of the Presidency of Bombay. Journal of the Indian Botanical Society 9: 151-174.

Dickie, G. 1876. Notes on algae collected by H.N. Moseley, M.A. of H.M.S. "Challenger", chiefly obtained in Tores Straits, coasts of Japan and Juan Fernandez. Journal of the Linnean Society of London, Botany 15: 446-455.

Egerod, L.E. 1952. An analysis of the Siphonous Chlorophycophyta with special reference to the Siphonocladales, Siphonales, and Dasycladales of Hawaii. University of California Publications in Botany 25: 327-367.

Faegri, K. \& Iversen, J. 1975. The principles of pollen analysis. Oxford, Blackwell Scientific Publication Ltd.

Guiry, M.D. \& Guiry, G.M. 2011. AlgaeBase. World-wide electronic publication, National University of Ireland, Galway. <http://www. algaebase.org > (Acesso em 10/05/2011).

Leliaert, F. \& Coppejans, E. 2004. Crystaline cell inclusions: a new diagnostic character in the Cladophorophyceae (Chlorophyta). Phycologia 43(2): 189-203.

Leliaert, F. \& Coppejans, E. 2007. Morphological re-assessment of the Boodlea composita-Phyllodictyon anastomosans species complex (Siphonocladales: Chlorophyta). Australian Systematic Botany 20: 161-185.

Leliaert, F., De Clerck, O., Verbruggen, H., Boedeker, C. \& Coppejans, E. 2007a. Molecular phylogeny of the Siphonocladales (Chlorophyta: Cladophorophyceae). Molecular Phylogenetics and Evolution 44: 1237-1256.

Leliaert, F; Huisman, J. \& Coppejans, E. 2007b. Phylogenetic position of Boodlea vanbosseae (Siphonocladales, Chlorophyta). Cryptogamie Algologie 28: 337-351.

Leliaert, F.; Millar, A.J.K.; Vlaeminck, C. \& Coppejans, E. 2007c. Systematics of the green macroalgal genus Chamaedoris Montagne (Siphonocladales), with an emended description of the genus Struvea Sonder. Phycologia 46: 709-725.

Leliaert, F.; Rosseau, F.; De Reviers, B. \& Coppejans, E. 2003. Phylogeny of the Cladophorophyceae (Chlorophyta) inferred from partial LSU rRNA gene sequences: is the recognition of separate order Siphonocladales justified? European Journal of Phycology 38: 233-246.

Leliaert, F.; Verbruggen, H.; Wysor, B. \& De Clerck, O. 2009. DNA taxonomy in morphologically plastic taxa: Algorithmic species delimitation in the Boodlea complex (Chlorophyta: Cladophorales). Molecular Phylogenetics and Evolution 53: 122-133.

Littler, D.S. \& Littler, M.M. 2000. Caribbean reef plants. An identification guide to the reef plants of the Caribbean, Bahamas, Florida and Gulf of Mexico. Washington, Offshore Graphics. 
Meñez, E.G. 1961. The marine algae of the Hundred Islands, Philippines. Philippine Journal of Science 90: 37-86.

Murray, G. 1889. On Boodlea, a new genus of Siphonocladaceae. Journal of the Linnean Society, Botany 25: 243-245.

Nunes, J.M.C. \& S.M.P.B. Guimarães. 2008. Novas referências de rodofíceas marinhas bentônicas para o litoral brasileiro. Biota Neotropica 8: 89-100.

Olsen-Stojkovich, J.L. 1986. Phylogenetic studies of genera in the Siphonocladales-Cladophorales complex (Chlorophyta). PhD dissertation. University of California, Berkeley.
Wynne, M.J. 2011. A Checklist of Benthic marine algae of the tropical and subtropical western Atlantic: third Revision. Nova Hedwigia Beihefte 140: 1-166.

Yasue, T. 1969. Histochemical identification of calcium oxalate. Acta Histochemica et Cytochemica 2: 83-95.

Yoneshigue, Y.; Boudouresque, C.F. \& Figueiredo, M.A.O. 1986. Flore algale marine de la Région de Cabo Frio, état de Rio de Janeiro (Brésil). 5 Sur Boodlea composita (Boodleaceae-Chlorophyta), Dictyota pardalis (Dictyotaceae-Phaeophyta) et Lophosiphonia cristata (RhodomelaceaeRhodophyta), espèces nouvelles pour la côte Brésilienne. Rickia 13: 17-27. 